\title{
Sur les Bathynella de Roumanie: B. (B.) boteai Serban, B. (B.) vaducrisensis N. Sp., B. (B.) plesai Serban et B. (B.) motrensis Serban
}

\author{
(Bathynellacea, Bathynellidae)
}

Deuxième partie

par

Eugène SERBAN

\author{
SUMMARY \\ On the Bathynella from Romania: B. (B.) boteai Serban, B. (B.) \\ vaducrisensis N. Sp., B. (B.) plesai Serban et B. (B.) \\ motrensis Serban (Bathynellacea, Bathynellidae). Part II
}

In the second part of the note a discussion is given dealing with the importance of different characters in the systematics of the genus Bathynella Vejdovsky and with species individualisation in the sub-genus Bathynella (Bathynella) Vejdovsky. We emphasize the differentiation of some elements of the VIIIth male pereiopodes, these appendages never having the same morphology in different species. Taking into account the structure of the apical end of the pennian anterior plates, we suggest to group separately boteai with vaducrisensis and plesai with ruffoi. The main conclusions of the recent studies on the European Bathynella are also given.

\section{DISCUSSION}

Les données que nous venons de présenter confirment nos premiers résultats à propos de la taxonomie des Bathynella de Roumanie (Serban, 1966, 1966a, 1971). A l'heure actuelle, la validité des espèces décrites est moins discutable, leurs diagnoses, plus complètes, se vérifiant sur plusieurs exemplaires. Toutefois, la connaissance fragmentaire des populations de Roumanie et surtout les données trop sommaires quant aux Bathynella d'Europe restent des facteurs objectifs impliquant à toute contribution certaines réserves.

Les nombreuses figures jointes au texte désirent mettre à la disposition des spécialistes un grand nombre de détails morphologiques si nécessaires dans l'étape actuelle de recherche, étape qui impose la solution d'une foule de

* Institut de Spéologie "Emile Racovitza", 9, rue Mihail Moxa, Bucarest, Roumanie. 
problèmes. Il suffit de rappeler que l'appartenance générique des "Bathynella" d'Asie n'a pas été encore élucidée et que les rares recherches concernant les représentants d'Amérique australe, d'Afrique et d'Australie sont loin d'être satisfaisantes. Ce manque de données empêche la mise en évidence de la diversification réelle des Bathynellidés sur les divers continents et rend impossible la connaissance des grandes voies évolutives du groupe. Il s'ensuit donc, que la large répartition de la famille soulève de nombreuses questions qui exigent des recherches approfondies et des efforts soutenus; forcément, le point de départ de toutes ces études ne peut être que le genre Bathynella, groupe dont la structure, la diversification et la répartition géographique doivent être connues le plus tôt possible.

\section{Valeur des divers caractères dans la systématique du genre Bathynella}

Dans ce qui suit on insistera surtout sur les questions intéressant le sous-genre Bathynella (Bathynella) dont les six espèces mieux décrites offrent un point de départ sûr dans l'analyse que nous nous proposons concernant la valeur taxonomique des caractères.

a. La plaque antérieure et la proéminence externe des péréiopodes VIII mâles (tableau 14). La partie apicale de la plaque antérieure, bien différente chez les espèces paranatans, boteai, plesai et motrensis, à morphologie identique chez $B$. (B.) boteai et $B$. (B.) vaducrisensis, d'une part, et chez $B$. (B.) motrensis et $B$. chappuisi, d'autre part, est peu différenciée chez $B$. (B.) plesai et $B$. (B.) ruffoi. Si dans les cas des espèces boteai et vaducrisensis ou plesai et ruffoi, représentants du sous-genre Bathynella (Bathynella), l'identité mentionnée suppose que chacun de ces deux couple de taxa appartient au même groupe d'espèces, la similitude frappante des plaques antérieures des $B$. (B.) motrensis et $B$. chappuisi suggère une autre interprétation, car B. chappuisi semble appartenir au sous-genre Bathynella (Lombardobathynella), taxon caractérisé par la présence d'un lobe parapénien (le quatrième lobe du complexe pénien) et par l'absence de la papille sur la plaque antérieure (Serban, 1973a; Serban et Delamare Deboutteville, 1974). Or, l'existence d'une plaque antérieure ayant la partie apicale structurée similairement chez deux espèces appartenant à deux sous-genres indique, à notre avis, le degré très réduit de la diversification de ce lobe dans le genre Bathynella; il n'est donc pas surprenant de trouver chez plusieurs espèces une plaque très semblable voire identique. Il est donc logique de penser que le manque de différenciation de la plaque chez plusieurs représentants du genre doit être compensé par le remaniement d'un autre niveau de la morphologie du pénis, de sorte que cet appendice, pris dans sa totalité, soit caractéristique de chaque espèce. Comme on a vu au cours des descriptions, la proéminence externe est l'un de ces niveaux.

En comparant la forme de la proéminence externe chez les diverses espèces du sous-genre Bathynella (Bathynella), il ressort que, sauf B. (B.) boteai, chez les autres cette formation a le bord arrondi. Même si dans le cas de $B$. (B.) motrensis s'individualise un angle peu proéminent dans la région où les parties verticale et horinzontale du bord se rencontrent, chez $B$. (B.) 
Tableau 14. Différenciation des caractères chez cing espèces de Bathynella de Roumanie

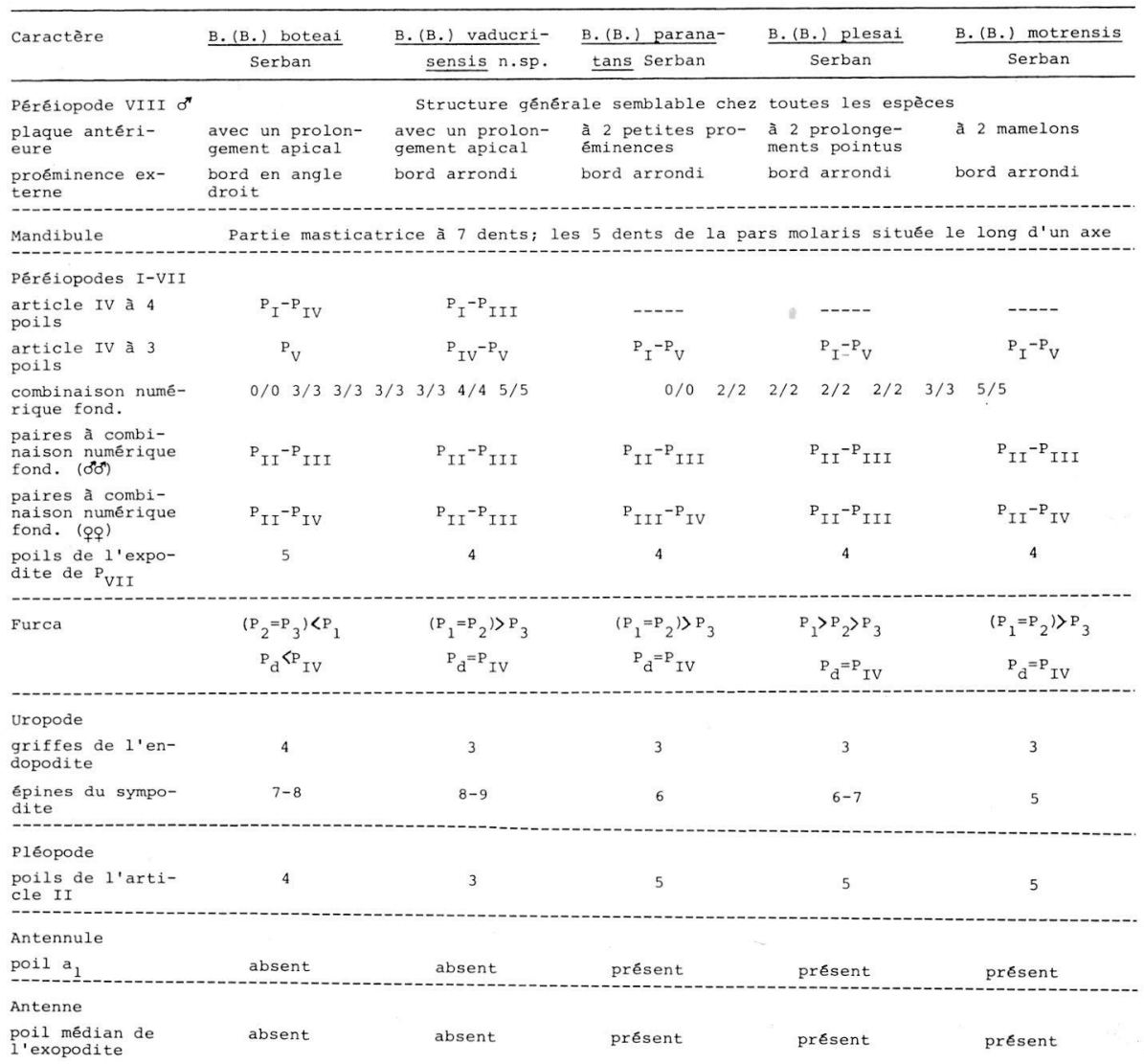

$\mathrm{P}_{\mathrm{I}}=$ përéiopode $\mathrm{I} ; \mathrm{P}_{1}=$ poil furcal $1 ; \mathrm{P}_{\mathrm{d}}=$ poil dorsal de la furca.

paranatans, B. (B.) plesai, B. (B.) vaducrisensis et B. (B.) ruffoi il nous a été impossibe de saisir des différences notables. A cette uniformité structurale de la proéminence externe chez les quatre espèces il faut également ajouter sa similitude chez $B$. chappuisi et $B$. (L.) lombardica (voir les fig. 7A, B in Serban et Delamare Deboutteville, 1974 et la fig. 9 in Serban, 1973a). En somme, de même que la plaque antérieure, la proéminence externe n'est pas toujours un élément spécifique. 
En considérant maintenant le couple de traits plaque antérieure + proéminence externe, on remarque: lorsque la partie apicale de la plaque est identique $-B$. (B.) boteai et $B$. (B.) vaducrisensis ou $B$. (B.) motrensis et $B$. chappuisi - c'est la proéminence qui est nettement différenciée et lorsque la proéminence a une forme identique $-B$. (B.) vaducrisensis, $B$. (B.) paranatans, $B$. (B.) plesai et $B$. (B.) ruffoi - c'est la plaque qui présente une structure spécifique. Vu l'appartenance de ces espéces à deux sous-genres, ainsi que leur degré d'affinité plus ou moins accusée, la modification compensatrice de la proéminence externe de $B$. (B.) boteai et de $B$. (B.) vaducrisensis (espèces très rapprochées et ayant les plaques antérieures identiques) prend une importance capitale dans la diversification de leurs pénis.

b. Mandibule (tableau 14). De même que la structure générale des péréiopodes VIII mâles, la morphologie de la partie masticatrice mandibulaire représente un caractère essentiel du genre Bathynella. Il ne s'agit pas seulement du nombre total de 7 dents, mais de la disposition des 5 dents de la pars molaris situées l'une après l'autre le long d'un seul axe. Il faut tenir compte de ce dernier trait, car dans le genre Sardobathynella Serban, cette pièce buccale est également munie de 7 dents, mais les 5 de la pars molaris sont autrement disposées; les 4 premières sont groupées deux par deux sur des plans parallèles à celui de la pars incisiva.

c. Chétotaxie des péréiopodes ambulatoires (tableau 14). Selon les caractéristiques les plus générales de la chétotaxie des péréiopodes ambulatoires, les cinq espèces de Roumanie peuvent être réparties en deux groupes (il ne s'agit pas de groupes à valeur taxonomique): le premier comprend $B$. (B.) boteai et $B$. (B.) vaducrisensis et le second, $B$. (B.) paranatans, $B$. (B.) plesai et $B$. (B.) motrensis. Dans le premier groupe les péréiopodes I portent entre 48 et 54 phanères, l'article endopodial IV des paires antérieures est pourvu de 4 poils et la combinaison numérique fondamentale, comprenant 42 phanères, a comme élément le plus fréquent le nombre de 3 poils $(0 / 03 / 3 \quad 3 / 3 \quad 3 / 3$ $3 / 34 / 45 / 5$ ). Dans le second groupe, les péréiopodes I ont entre 40 et 44 poils, l'article endopodial IV des paires I-V est muni de 3 poils et la combinaison numérique, composée de 32 phanères, a comme élément le plus fréquent le nombre de 2 poils $(0 / 02 / 22 / 22 / 22 / 23 / 35 / 5)$. Les différences numériques existant entre les deux groupes quant à la chétotaxie des péréiopodes I-IV se réduisent visiblement dans le cas des péréiopodes $\mathrm{V}$, tandis que sur les paires VI et VII la chétotaxie est presque identique chez toutes les cinq espèces. Il en ressort, qu'à l'opposé de la plupart des appendices du tronçon péréial typique (les 5 premiers métamères; Serban, 1973c) ayant un nombre de poils bien différent chez les deux groupes, les appendices ambulatoires du tronçon génital du péréion (les 3 derniers métamères) ont une chétotaxie très semblable.

En soulignant encore une fois que la combinaison numérique fondamentale $0 / 03 / 33 / 33 / 33 / 34 / 45 / 5$ est propre aux deux espèces du premier groupe et que la combinaison $0 / 02 / 22 / 22 / 22 / 23 / 35 / 5$ est présente chez toutes les trois espèces du second groupe, il est à relever également que la chétotaxie, prise dans sa totalité, n'est jamais identique chez les espèces d'un 
groupe. Dans cette différenciation, considérée séparément pour chaque groupe, certains éléments y jouent un rôle important. Ceux-ci sont: la chétotaxie des péréiopodes I des deux sexes qui n'est pas identique chez les divers taxa et l'existence de la combinaison numérique fondamentale sur un nombre variable des péréiopodes (femelles) (le dernier éléments implique seulement les paires II, III et IV). A ces traits on peut ajouter la chétotaxie des péréiopodes $\mathrm{V}$ qui peut être soit différente chez les deux sexes $-B$. (B.) boteai, B. (B.) paranatans et $B$. (B.) motrensis - , soit identique $-B$. (B.) vaducrisensis et $B$. (B.) plesai.

Parmi les 5 espèces, c'est chez $B$. (B.) motrensis que la différenciation de la chétotaxie est des plus intéressantes, la combinaison numérique des péréiopodes $I$ étant, chez les mâles et les femelles, identique à la combinaison de $B$. (B.) plesai et respectivement, de B. (B.) paranatans. A ce type particulier d'individualisation dû à une chétotaxie des paires I qui combine, selon le sexe, la chétotaxie des deux espèces s'ajoute, chez les femelles, la présence de la combinaison numérique fondamentale sur 3 paires de péréiopodes, caractéristique absente chez les deux autres espèces du groupe, mais présente chez les femelles de $B$. (B.) boteai. Cette manière de différenciation nous laisse croire que dans le genre Bathynella la chétotaxie ayant comme combinaison numérique fondamentale $0 / 02 / 22 / 22 / 22 / 23 / 35 / 5$ doit être tenue pour une chétotaxie limite et la moins riche possible. Autrement dit, on ne peut pas s'attendre à une réduction plus accentuée de cette combinaison intimement liée au nombre de poils de tous les 5 premiers péréiopodes et, en conséquence, il sera très probable de trouver chez plusieurs espèces une chétotaxie identique sur tous les appendices; selon nous, le nombre réduit de poils propre à la chétotaxie en question ne permet pas sa différenciation dans un très grand nombre de possibilités.

Dans les observations ci-dessus, nous avons tenu compte de la variabilité numérique des poils et du nombre assez réduit des exemplaires étudiés pour chaque espèce. Toutefois, nos résultats exigent des études plus poussées, les seules qui permettraient d'élucider d'une manière plus sûre la valeur taxonomique de la chétotaxie des péréiopodes ambulatoires.

d. Chétotaxie de la furca (tableau 14). Selon les rapports les plus généraux entre la longueur des poils 1, 2 et 3, les furca des espèces de Bathynella se groupent de la façon suivante:

1) furca ayant les poils 1 et 2 égaux et le poil 3 plus court: $B$. (B.)paranatans, B. (B.) motrensis, B. (B.) ruffoi, B. (B.) vaducrisensis et B. chappuisi;

2) furca ayant les poils 2 et 3 de la même longueur et le poil 1 plus long: B. (B.) boteai;

3) furca ayant les poils 1, 2 et 3 de longueurs qui décroissent graduellement: B. (B.) plesai;

4) furca ayant les poils 1 et 3 de taille égale et le poil 2 plus long: $B$. (B.) scythica;

5) furca ayant les poils 1,2 et 3 de la même longueur: $B$. (L.) lombardica.

A notre avis, ces cinq catégories de chétotaxie de la furca épuisent toutes les possibilités quant aux rapport généraux entre les poils 1, 2 et 3 dans le genre 
Bathynella. Nous ne pensons pas que les poils 1 et 2 puissent être plus courts que le poil 3 ou que le poil 1 soit si réduit que celui de Antrobathynella stammeri.

En nous rapportant à la taille du poil le plus développé dans les 5 types de furca, il est à souligner que sa longueur n'est jamais trop accusée par rapport à celle des autres poils (il s'agit des poils $1,2,3$ et 4). Nous insistons sur cette caractéristique car chez B. natans sensu Chappuis, 1915, de Bâle, le poil 2 est exceptionnellement allongé, trait tenu plusieurs décennies pour une différence importante entre B. natans et B. chappuisi. Cet allongement du poil furcal 2, qui se rencontre fréquemment dans les genres Gallobathynella, Meridiobathynella et Pseudobathynella, nous ne l'avons pas trouvé chez les adultes de Bathynella. D'ailleurs, l'opinion de P. A. Chappuis selon laquelle le matériel de Bâle appartiendrait à B. natans n'est plus valable (voir Pseudobathynella helvetica in Serban et Delamare Deboutteville, 1974).

Etant donné les cinq espèces dont les poils 1 et 2 sont de taille égale, nous avons cherché d'autres éléments pouvant caractériser la furca de chaque taxon. D'après la longueur du poil 4 et du poil dorsal, la furca de B. chappuisi se distingue nettement de celle des autres espèces, car le poil dorsal, de la même longueur que le bras furcal, est plus court que le poil 4, tandis que chez $B$. (B.) paranatans, B. (B.) motrensis, B. (B.) ruffoi et B. (B.) vaducrisensis, le poil dorsal, bien plus court que le bras furcal, est de la même longueur que le poil 4 . Une séparation nette, selon la chétotaxie de la furca, de ces 4 dernières n'est pas possible.

e. Chétotaxie des uropodes (tableau 14). Les espèces de Bathynella portent de 5 (B. (B.) motrensis) à 9 épines (B. (B.) vaducrisensis) sur le sympodite des uropodes et de 3 (B. (B.) paranatans, $B$. (B.) motrensis, $B$. (B.) plesai, B. (B.) ruffoi, B. (B.) scythica, B. (L.) lombardica) à 4 griffes sur l'endopodite (B. (B.) boteai, B. chappuisi) A notre avis, ces nombres représentent des limites numériques caractérisant le genre.

Chez la majeure partie des représentants on trouve 7-6 épines et 3 griffes, ce qui diminue considérablement la signification taxonomique des uropodes. Le nombre des griffes ou celui des épines ne doit pas être pris comme un critère prouvant l'affinité plus ou moins étroite des divers taxa; l'espèce boteai, ayant 4 griffes, est plus éloignée de $B$. chappuisi que de $B$. (B.) vaducrisensis, bien que la première ait le même nombre de griffes et que la seconde n'en ait que 3. Il n'existe pas aussi de corrélation directe entre les nombres des deux types de phanères: $B$. (B.) boteai et $B$. chappuisi à 4 griffes, ont respectivement 8 et 6 épines, tandis que $B$. (B.) vaducrisensis, $B$. (B.) plesai, $B$. (B.) ruffoi et $B$. (B.) motrensis à 3 griffes en ont respectivement 8-9, 6-7 et 5 .

Afin de mieux saisir la diversification des uropodes dans le genre, nous avons tenu compte des caractéristiques suivantes: nombre de griffes + nombre d'épines + longueur de la griffe distale par rapport à celle de l'endopodite + longueur de la griffe distale par rapport aux autres deux ou trois griffes.

f. Chétotaxie des pléopodes (tableau 14). La chétotaxie de l'article apical de ces appendices est la seule à se diversifier chez certaines espèces. On trouve 3 (B. (B.) vaducrisensis), 4 (B. (B.) boteai, B. (B.) ruffoi) et 5 poils 
(B. (B.) paranatans, B. (B.) plesai, B. (B.) motrensis et B. chappuisi); leur répartition est la suivante: 2 sont toujours situés à la partie apicale de l'article, le troisième se trouve sur le bord dorsal (bord opposé à la proéminence de l'article proximal qui porte un poil), le quatrième s'insère sur le bord ventral et le cinquième sur le bord dorsal, du côté proximal du troisième poil. On a commencé la présentation des poils avec les deux apicaux car ils sont toujours présents. Généralement, le nombre des poils des pléopodes a une valeur taxonomique d'ordre spécifique.

g. Péréiopodes VIII femelles. Contrairement aux péréiopodes VIII mâles, les péréiopodes VIII femelles n'ont pas, en général, une structure spécifique. Dans les cas de $B$. (B.) boteai et $B$. (B.) motrensis, la position des poils des bords de l'exopodite et respectivement l'allongement visible de cet article sont des caractère différentiels.

h. Antennule (tableau 14). La seule différence à signaler chez les espèces de Roumanie est l'absence (B. (B.) boteai et $B$. (B.) vaducrisensis) ou la présence (B. (B.) paranatans, B. (B.) plesai et $B$. (B.) motrensis) du poil proximal de la face latéro-interne de l'article basal du pédoncule.

i. Antenne (tableau 14). Cet appendice, un peu plus court ou égal à l'antennule - sous-genre Bathynella (Bathynella) -, s'allonge visiblement chez B. (L.) lombardica. La ramification apicale de l'organe sensoriel peut être différente chez certaines espèces. Le poil médian de l'exopodite n'est pas développé chez $B$. (B.) boteai, B. (B.) vaducrisensis et $B$. (B.) ruffoi. Utiles à la taxonomie peuvent être, également, les rapports de longueur entre les articles endopodiaux II et IV ou ceux entre l'exopodite et l'article endopodial III.

Nous n'insisterons pas sur les différences spécifiques des maxillules et des maxilles.

\section{Sur l'individualisation et les affinités des espèces du sous-genre Bathynella (Bathynella)}

En partant des données que nous venons de présenter et tout spécialement de la discussion sur la valeur taxonomique des caractères, on arrive aux observations d'ordre général qui suivent:

- parmi les espèces étudiées, il n'en existe pas une dont l'individualisation se réalise au niveau de tous les appendices, de sorte que son stock général de caractères ne comprenne pas des éléments ayant la même morphologie chez plusieurs représentants du sous-genre. Ceci prouve l'étroite affinité de ces taxa et leur diversification dans le cadre du sous-genre par l'intermédiaire d'un jeu d'échange de caractères (Serban, 1973a, 1973b). Cet échange détermine des rapports morphologiques différents entre les espèces et donne aux divers traits une signification taxonomique variable. On rencontre des situations où certains caractères présentent une différenciation saillante et d'autres où ces mêmes caractères ont une morphologie très proche ou identique. Si l'on compare, par exemple, la furca, l'uropode, les péréiopodes ambulatoires et le pléopode de $B$. (B.) boteai et de $B$. (B.) paranatans, on voit que leur chétotaxie est nettement différenciée; mais si l'on essaye de séparer les espèces 
paranatans et plesai ou paranatans et motrensis selon les mêmes critères, on se rend compte que la plupart de ces appendices ont une valeur spécifique réduite ou nulle. Il est aisé de saisir aussi que la diversification des péréiopodes VIII mâles n'est pas de la même intensité chez tous les représentants: il est incontestable que chez $B$. (B.) boteai et B. (B.) paranatans cette diversification est bien plus prononcée que chez $B$. (B.) boteai et $B$. (B.) vaducrisensis ou chez B. (B.) paranatans et B. (B.) motrensis. Ces rapports variables entre les divers taxa du sous-genre Bathvnella (Bathynella) suggèrent des relations de parenté différentes et, par conséquent, l'existence de plusieurs groupes d'espèces.

- la diversification générale des caractères révèle un assez faible degré, leur potentialité structurale étant réduite dans le sous-genre (voir la furca, l'uropode, le pléopode, la forme de la proéminence externe du péréiopode VIII mâle). Ce degré, qui ne permet pas que chaque espèce s'individualise par des modifications morphologiques inédites des divers appendices, se trouve compensé par le remaniement du stock général de caractères; généralement l'ensemble des traits prend l'aspect d'une combinaison - différente pour chaque espèce - de certains caractères qui ont la même structure chez d'autres représentants du genre aussi. Les péréiopodes VIII mâles font exception restant caractéristiques de chaque taxon.

- étant donné les affinités entre les cinq espèces de Roumanie, on peut les séparer en deux groupes: d'une part $B$. (B.) boteai et $B$. (B.) vaducrisensis et d'autre part, B. (B.) paranatans, B. (B.) motrensis et B. (B.) plesai (tableau 14). Mais, si l'on considère aussi $B$. (B.) ruffoi d'Italie, on voit que $B$. (B.) plesai s'avère plus proche de ce représentant que de $B$. (B.) paranatans et de B. (B.) motrensis. Il ressort donc, que parmi les six Bathvnella en question on distingue en toute certitude deux groupes d'espèces: le groupe boteai qui réunit $B$. (B.) boteai et $B$. (B.) vaducrisensis et le groupe plesai comprenant B. (B.) plesai et B. (B.) ruffoi. Quant aux B. (B.) paranatans et B. (B.) motrensis, il nous est difficile de décider s'ils appartiennent ou non au même groupe en raison de la dissemblance de leurs plaques antérieures et du dessin argyrophile de la cuticule dont les sclérites pléonaux n'ont pas la même forme (voir Pl. XIX, XX, LXII, in Serban, 1972). Bien que nous ne sachions pas quelle est la valeur taxonomique de ce dernier caractère, il faut préciser que la différenciation des dessins argyrophiles de $B$. (B.) vaducrisensis et de $B$. (B.) paranatans correspond à l'appartenance de ces espèces à des groupes différents.

Quoique nos connaissances concernant le sous-genre Bathvnella (Bathynella) puissent être tenues pour trop sommaires pour pouvoir grouper les espèces, nous croyons absolument nécessaire d'aborder la discussion de ce problème. Le caractère complexe de la diversification dans le sous-genre impose - même à ce stade des recherches - l'analyse de toutes les décisions prises. N'oublions pas que c'est pour la troisième fois que l'on reprend l'étude taxonomique de Bathynella (Chappuis, 1914, 1915;Jakobi, 1954) et que jusqu'à présent, 90 ans après la découverte de ce taxon, on n'est pas arrivé à des résultats satisfaisants. 
Dans les considérations les plus générales sur la diversification évolutive dans la famille des Bathynellidae, nous sommes parti de l'hypothèse suivant laquelle ce processus se manifeste inégalement au niveau du péréiopode VIII mâle (caractère primordial) et des autres appendices (caractères secondaires) (Serban, 1973a, 1973b). Tenant compte du degré de différenciation de ces deux types de caractères dans les divers taxa, nous pensons qu'ils présentent une différenciation compensatrice: lorsque les pénis conservent une structure peu modifiée, ce sont les caractères secondaires qui se diversifient fortement (sous-famille des Gallobathynellinae) et lorsque les pénis sont bien différents, ce sont les caractères secondaires qui conservent une morphologie peu modifiée (voir les rapports entre Bathvnella-Austrobathynella, et BathynellaAntrobathynella). Précisons que la différenciation très accusée des pénis n'exclue point la présence de certains caractères secondaires à structure typique pour un taxon donné (voir la mandibule dans le genre Bathynella) et que dans l'analyse du processus en question il faut tenir compte surtout du facteur qui domine l'individualisation d'un groupe.

La différenciation compensatrice dans le sous-genre Bathvnella (Bathynella) a été mise en évidence lors de l'étude de $B$. (B.) ruffoi, la première espèce qui nous a permis de saisir l'existence du premier groupe d'espèces, le groupe plesai (Serban, 1973a). Revenons brièvement sur ce problème.

Selon les détails de la morphologie du péréiopode VIII mâle $B$. (B.) ruffoi se rapproche de $B$. (B.) plesai, car ils sont les seuls à avoir la plaque antérieure munie de deux prolongements. C'est donc la similitude frappante de la plaque antérieure qui nous a déterminé de réunir les deux taxa dans un groupe d'espèces. En ce qui concerne les caractères secondaires de $B$. (B.) ruffoi, on voit que sa furca est identique à celles de $B$. (B.) paranatans et de $B$. (B.) motrensis, que la combinaison numérique fondamentale de la chétotaxie des péréiopodes est la même chez $B$. (B.) paranatans, $B$. (B.) motrensis et $B$. (B.) plesai, que les 5 poils de l'exopodite des péréiopodes VII rappellent $B$. (B.) boteai, que le nombre d'épines du sympodite des uropodes est similaire à celui de $B$. (B.) plesai et que la chétotaxie de l'endopodite des uropodes n'est pas significative (on trouve trois griffes chez cinq espèces); la présence de l'épipodite respiratoire sur les péréiopodes I et l'absence du poil médian sur l'exopodite antennaire sont des traits qui rappellent les espèces paranatans et respectivement boteai et vaducrisensis. Cette combinaison de caractères secondaires chez B. (B.) ruffoi, comprenant des éléments qui se retrouvent chez les autres représentants du sous-genre, individualise clairement l'espèce par rapport au B. (B.) plesai, la plupart de ses appendices ayant une morphologie différente (voir furca, la présence ou l'absence du poil médian de l'exopodite antennaire et de l'épipodite respiratoire sur les péréiopodes I, la chétotaxie des pléopodes). En ce qui concerne l'individualisation de $B$. (B.) ruffoi par rapport aux autres représentants du sous-genre, elle est nettement marquée autant par la plaque antérieure des péréiopodes VIII mâles que par le stock de caractères secondaires pris dans sa totalité. La différenciation d'ordre spécifique des péréiopodes VIII mâles des deux espèces du groupe plesai se réalise au niveau de 
la plaque antérieure même, par la taille et la forme différente des prolongements. En somme, à la structure générale semblable des plaques antérieures et à la faible différenciation de leurs parties apicales, éléments qui marquent les affinités immédiates de $B$. (B.) plesai et de $B$. (B.) ruffoi et leur appartenance au même groupe d'espèces, correspond une différenciation accusée des caractères secondaires se manifestant par une combinaison - différente pour chacun - de certains caractères ayant la même morphologie chez d'autres espèces du sous-genre.

Cette manière de concevoir la diversification évolutive dans le sous-genre Bathynella (Bathynella) - fondée initialement sur l'étude de $B$. (B.) plesai et $B$. (B.) ruffoi - se vérifie en bonne mesure dans le cas du groupe boteai.

Dès le début il faut retenir que, pareillement au groupe plesai, dans le groupe boteai c'est toujours la plaque antérieure des péréiopodes VIII mâles qui démontre l'étroite parenté des deux espèces. Mais cette fois-ci, la différenciation spécifique des pénis n'implique pas cette formation mais les proéminences externes à bord en angle droit $(B .(B$. $)$ boteai) ou semi-circulaire (B. (B.) vaducrisensis). A la morphologie des péréiopodes VIII mâles s'ajoute la chétotaxie des péréiopodes ambulatoires et surtout la combinaison numérique fondamentale $0 / 03 / 33 / 33 / 33 / 34 / 45 / 5$. Chez $B$. (B.) vaducrisensis le nombre plus réduit de poils et de paires de péréiopodes qui portent 4 phanères apicaux suggère une tendance de réduction de cette chétotaxie vers la chétotaxie dont la combinaison numérique fondamentale est $0 / 02 / 2$ $2 / 22 / 22 / 23 / 35 / 5$. Il serait intéressant de voir si chez d'autres espèces de ce groupe, qu'il reste à découvrir, cette tendance sera plus accentuée. Quant aux autres caractères communs au deux espèces, il est à mentionner l'absence du poil médian de l'exopodite antennaire et de l'épipodite respiratoire sur les péréiopodes I et le nombres des épines du sympodite des uropodes, toujours plus grand que 7 .

Parmi les caractères différentiels les plus saillants des deux espèces, il est à noter la chétotaxie de la furca, de l'endopodite des uropodes, de l'exopodite des péréiopodes VII et des pléopodes; dans le cas de $B$. (B.) vaducrisensis, les trois premiers traits rappellent les espèces motrensis et paranatans.

En conclusion, de même que dans le groupe plesai, les deux espèces du groupe boteai se distinguent entre elles par une faible différenciation des péréiopodes VIII mâles et par une série de caractères secondaires à structure bien différente. L'individualisation du groupe même se réalise, outre les péréiopodes VIII mâles, par plusieurs caractères secondaires (chétotaxie des péréiopodes, absence du poil médian sur l'exopodite antennaire, nombre élevé des épines sur le sympodite des uropodes). En même temps, chez les deux espèces on rencontre des caractères similaires à ceux des autres représentants du genre.

Avant de passer aux conclusions qui ressortent de cette note, nous présenterons les clefs de détermination pour les mâles et les femelles des six espèces. 
Clef de détermination des six espèces du sous-genre Bathynella (Bathynella) (mâles)

1 - Plaque antérieure des péréiopodes VIII mâles sans formations apicales

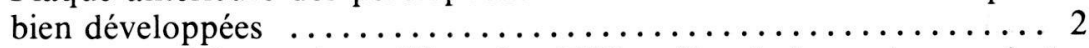

- Plaque antérieure des périopodes VIII mâles à formations apicales

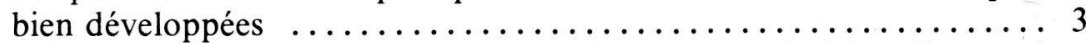

2 - Plaque antérieure à 2 petites proéminences; $P_{1}$ à épipodite respira-

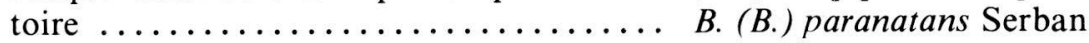

- Plaque antérieure à 2 mamelons; $\mathrm{P}_{\mathrm{I}}$ sans épipodite respiratoire ..... $\ldots \ldots \ldots \ldots \ldots \ldots \ldots \ldots \ldots$ B. (B.) motrensis Serban

3 - Plaque antérieure avec un prolongement apical et rostral bien développé; combinaison numérique fondamentale de la chétotaxie des $\mathrm{P}_{\mathrm{I}}-\mathrm{P}_{\mathrm{VII}}=0 / 03 / 33 / 33 / 33 / 34 / 45 / 5 \ldots \ldots \ldots \ldots \ldots \ldots \ldots$

- Plaque antérieure à 2 prolongements apicaux; combinaison numérique fondamentale de la chétotaxie des $P_{1}-P_{V I I}=\begin{array}{lllll}0 / 0 & 2 / 2 & 2 / 2 & 2 / 2 & 2 / 2\end{array}$

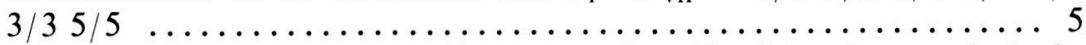

4 - Proéminence externe des péréiopodes VIII mâles à bord en angle droit; uropode à 4 griffes endopodiales .............. (B.) boteai Serban

- Proéminence externe des péréiopodes VIII mâles à bord semi-circulaire; uropodes à 3 griffes endopodiales ........ B. (B.) vaducrisensis n. sp.

5 - Prolongements de la plaque antérieure de taille différente, pointus; $\mathrm{P}_{1}$ sans épipodite respiratoire ............... (B.) plesai Serban

- Prolongements de la plaque antérieure de taille semblable, à région apicale arrondie; $\mathrm{P}_{\mathrm{I}}$ à épipodite respiratoire .... B. (B.) ruffoi Serban

\section{Clef de détermination des six espèces du sous-genre \\ Bathynella (Bathynella) (femelles)}

1 - Combinaison numérique fondamentale de la chétotaxie des $\mathbf{P}_{\mathrm{I}}-\mathbf{P}_{\mathrm{VII}}$

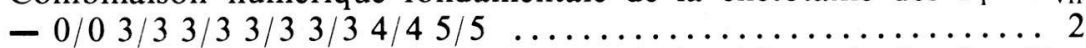

- Combinaison numérique fondamentale de la chétotaxie des $\mathrm{P}_{\mathrm{l}}-\mathrm{P}_{\mathrm{VII}}$ $=0 / 02 / 22 / 22 / 22 / 23 / 35 / 5 \ldots \ldots \ldots \ldots \ldots \ldots \ldots \ldots \ldots \ldots \ldots$

2 - Uropode à 4 griffes endopodiales; article distal du pléopode muni de 4 poils; sympodite de l'uropode à 7-8 épines ... B. (B.) boteai Serban

- Uropode à 3 griffes endopodiales; article distal du pléopode muni de 3 poils; sympodite de l'uropode à $8-9$ épines $\ldots \ldots \ldots \ldots \ldots \ldots \ldots$

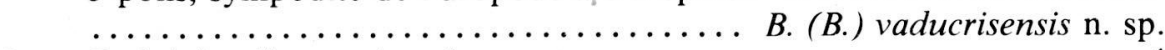

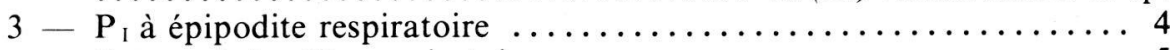

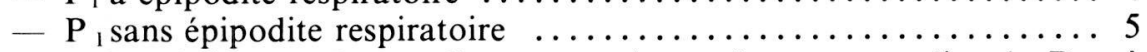

4 - Poil médian de l'exopodite antennaire, présent; exopodite de $\mathrm{P}_{\mathrm{VII}}$ à 4 poils; article distal du pléopode à 5 poils ....B. (B.) paranatans Serban

- Poil médian de l'exopodite antennaire, absent; exopodite de $\mathrm{P}_{\mathrm{VI}}$ à 5 poils; article distal du pléopode à 4 poils ......B. (B.) ruffoi Serban

5 - Sympodite de l'uropode à 5 épines; furca: $\left(\mathrm{P}_{1}=\mathrm{P}_{2}\right)>\mathrm{P}_{3}$; poil médian de l'article IV des péréiopodes pas plus robuste que les autres ...... 
- Sympodite de l'uropode à 7 épines; furca: $\mathrm{P}_{1}>\mathrm{P}_{2}>\mathrm{P}_{2}$; poil de l'article IV des péréiopodes bien plus robuste que les autres $\ldots \ldots \ldots \ldots \ldots$.

\section{CONCLUSIONS}

Les nouvelles données de cette note viennent compléter notre premier essai concernant la systématique, la diversification et l'individualisation des espèces du genre Bathynella (Serban, 1971). Sans avoir la prétention que les résultats et les opinions présentés soient irréfutables, nous croyons, toutefois, qu'ils constituent un point de départ utile aux futures recherches.

Par la présente contribution nous désirons offrir de nouveaux arguments en faveur de nos premiers résultats concernant les Bathvnella de Roumanie (Serban, 1966, 1966a, 1971, 1972) et de la validité de la nouvelle conception taxonomique que nous avons proposée. Si l'on considère nos contributions sur les Bathynella d'Europe (Serban, 1973a; Serban et Delamare Deboutteville, 1974) ou les études intéressant les autres genres de la famille (Serban, Coineau et Delamare Deboutteville, 1971, 1972; Serban, 1973, 1973b; Delamare Deboutteville et Serban, 1973; Serban et Coineau, 1975), on peut conclure que la nouvelle manière de concevoir la systématique des $\mathrm{Ba}$ thynellidae a permis la mise en évidence des éléments structuraux qui sont "assimilés" par la diversification du groupe et l'estimation plus précise de leur valeur taxonomique.

Parmi les traits les plus importants quı caractérisent le genre Bathynella, l. est à retenir la structure générale des péréiopodes VIII mâles et de la partie masticatrice mandibulaire. La constance morphologique de ces traits chez toutes les espèces permet d'identifier indubitablement n'importe quel représentant du genre.

Si la structure générale des péréiopodes VIII mâles caractérise le genre Bathynella, si l'existence de 3 ou 4 lobes dans le complexe pénien est propre aux sous-genres Bathynella (Bathynella) et respectivement Bathynella (Lombardobathynella), si la forme de la plaque antérieure ou celle de la proéminence externe, chacune prise à part, peut être ou non un trait séparant les espèces, ces deux derniers caractères, pris ensemble, révèlent en toute certitude la diversification d'ordre spécifique des péréiopodes VIII mâles.

En dehors des péréiopodes VIII mâles, ayant une morphologie qui indique tous les niveaux de la diversification dans le genre et de la partie masticatrice mandibulaire à structure uniforme chez tous les représentants, les autres caractères jouent un rôle surtout dans l'individualisation des espèces. Généralement, chaque espèce comporte un stock de caractères secondaires qui ne diffère pas totalement de celui des autres représentants. Cette manière d'individualisation ne met pas en doute la validité des taxa décrits, car c'est l'ensemble de tous les caractères, la différenciation compensatrice des caractères primordial et secondaires et la combinaison différente chez les divers taxa des caractères secondaires qui marquent la diversification. L'étude poussée des quatre espèces faisant l'objet de cette note, de même que celle de $B$. (B.) 
paranatans Serban (Serban, 1972), révèlent le rôle de la plupart des caractères secondaires dans le processus de la diversification; à présent, même la chétotaxie de l'article basal de l'antennule, de l'exopodite antennaire, des péréiopodes ambulatoires et des pléopodes s'avère utile dans les considérations d'ordre taxonomique.

D'après la structure de la plaque antérieure des péréiopodes VIII mâles, il a été possible d'identifier dans le sous-genre Bathvnella (Bathynella) deux groupes d'espèces: le premier, le groupe boteai, comprend B. (B.) boteai Serban et $B$. (B.) vaducrisensis $\mathrm{n}$. sp. ayant la plaque antérieure munie d'un prolongement apical et rostral; le second, le groupe plesai, est représenté par B. (B.) plesai Serban et $B$. (B.) ruffoi Serban dont la plaque antérieure porte deux prolongements apicaux. Quant aux espèces $B$. (B.) paranatans Serban et B. (B.) motrensis Serban, il nous est impossible, pour le moment, de décider s'ils appartiennent ou non au même groupe d'espèces.

Les connaissances trop fragmentaires des espèces du genre Bathynella ne nous permettent pas de pousser plus loin l'étude du problème de la diversification dans le groupe.

Rappelons encore une fois que les Bathvnelles d'Asie et d'Australie n'appartiennent pas au genre Bathynella, ce qui impose leur révision.

\section{RÉSUMÉ}

Dans cette seconde partie de la note on discute à propos de la valeur de certains caractères dans la systématique du genre Bathvnella Vejdovsky et de l'individualisation des espèces dans le sous-genre Bathvnella (Bathynella) Vejdovsky. On insiste sur la différenciation de certains éléments structuraux des péréiopodes VIII mâles, appendices qui ne présentent jamais une morphologie identique chez plusieurs espèces. En tenant compte de la structure de la partie apicale des plaques antérieures péniennes, on propose le groupement des espèces boteai et vaducrisensis, d'une part et plesai et ruffoi, d'autre part, en deux groupes. La note s'achève par les conclusions les plus importantes qui résultent des études récentes sur les Bathvnella d'Europe.

\section{BIBLIOGRAPHIE}

BARTOK, P. 1944. A Bathynella chappuisi fejlödés morphológiaja. Acta Sci. Math. Nat. Univ. Francisco-Josephina, Koloszvar, XII, 1-46.

BOTOSANEANU, L. 1959. Bathynellacea. Fauna R.P.R., Crustacea, IV, 5, 1-35.

BOTOSANEANU, L. \& DAMIAN, A. 1956. Bathynella (Crust. Syncarida) dans l'eau des conduites de Bucarest et de Constantza. Acta Soc. Zool. Bohemoslovenicae, XX, 4, 358-362.

CHAPPUIS, P. A. 1914. Über die systematische Stellung von Bathynella natans Vejdovsky. Zool. Anz., XLIV, 45-46.

CHAPPUIS, P. A. 1915. Bathynella natans und ihre Stellung im System. Zool. Jahrb., XL, $147-176$.

CHAPPUIS, P. A. 1924-1925. Sur les Copépodes et les Syncarides des eaux souterraines de Cluj et des monts Bihors. Soc. de stiinte din Cluj, II, 4, 157-158. 
CHAPPUIS, P. A. 1944. Die Grundwasserfauna der Körös und des Szamos. Math. Term. Közl. Vonat. Mag. Tudomanyos Akademia, XL, 2, 1-44.

CHAPPUIS, P. A. 1948. Copépodes, Syncarides et Isopodes des eaux phréatiques de Suisse. Rev. Suisse de Zool., LV, 30, 549-566.

DELACHAUX, TH. 1920. Bathynella chappuisi $\mathrm{n}$. sp. une nouvelle espèce de Crustacé cavernicole. Bull. Soc. Sci. Nat. Neuchâteloise, XLIV, 1-20.

Delamare DEBOUTTEVILLE, CL. 1960. Présence d'un Syncaride d'un genre nouveau dans les eaux interstitielles des lacs de la Patagonie andine et remarques biogéographiques. C. R. Acad. Sci., CCLI, 1038-1039.

DELAMARE DEBOUTTEVILLE, CL. \& CHAPPUIS, P. A. 1954. Les Bathynelles de la France et d'Espagne. Arch. Zool. exp. et gén., XCI, 1, 51-73.

DelamaRE DEBOUTTEVILlE, CL. \& ROLAND, CH. 1963. Syncarides. Austrobathynella patagonica Delamare. In Biologie de l'Amérique Australe, II, 55-62.

DELAmARE DEBOUTTEVILle, CL. \& SERBAN, E. 1973. A propos du genre Austrobathynella (Malacostraca Bathynellacea). Livre du cinquantenaire de l'Institut de Spéologie "Emile G. Racovitza". Ed. Acad. de la R.S.R., 175-198.

HUSMANN, S. 1968. Okologie, Systematik und Verbreitung zweier in Norddeutschland simpatrisch lebender Bathynella-Arten (Crustacea Syncarida). Int. J. Speleol., İII, $1+2$ (1976), 111-145.

JAKOBI, H. 1954. Biologie, Entwicklungsgeschichte und Systematik von Bathynella natans Vejd. Zool. Jb. (Syst), LXXXIII, 1-2, 1-184.

NOODT, W. 1965. Natürliches System und Biogeographie der Syncarida (CrustaceaMalacostraca). Gewässer und Abwässer, 37/38 (1964) 77-187.

NOODT, W. 1971. Die Bathynellacea Chiles. Gewässer und Abwässer. 50/51. 41-65.

NOODT, W. 1972. Brasilianische Grundwasser-Crustacea, 2. Nannohathvnella, Leptobathynella und Parabathynella aus der Serra do Mar von Sao Paulo (Malacostraca, Syncarida). Crustaceana, XXIII, 2, 152-164.

PLESA, C. 1967. Recherches sur la périodicité de reproduction chez les cavernicoles. Spelunca Mém. 5, 295-299.

SCHMINKE, H. K. 1973. Evolution, System und Verbreitungsgeschichte der Familie Parabathynellidae (Bathynellacea, Malacostraca). Mikrofauna Meeresboden, XXIV, 1-192.

SCHMINKE, H. K. \& WELLS, J. B. J. 1974. Nannohathvnellea africana sp. n. and the zoogeography of the family Bathynellidae (Bathynellacea, Malacostraca). Arch. Hydrobiol., LXXIII, 1, 122-129.

SERBAN, E. 1966. Contribution à l'étude de Bathvnella d'Europe; Bathvnella natans Vejd. un dilemme à résoudre. Int. J. Speleol., II, 115-132.

SERBAN, E. 1966a. Nouvelles contributions à l'étude de Bathynella (Bathvnella) natans Vejd. et Bathynella (Antrobathynella) stammeri Jakobi. Int. J. Speleol., II, 208-221.

SERBAN, E. 1970. A propos du genre Bathvnella Vejdovsky (Crustacea Syncarida). Livre du centenaire Emile G. Racovitza. Ed. Acad. de la R.S.R., Bucarest, 265-273.

SERBAN, E. 1971. Quatre nouvelles Bathynella (Crustacea Syncarida) de Roumanie; de nouveau sur le "Dilemme Bathynella natans Vejd.". Int. J. Speleol., III, $3+4$, 225-240.

SERBAN, E. 1972. Bathynella (Podophallocarida Bathynellacea). Trav. Inst. Spéol. ¿Emile Racovitza", XI, 11-224.

SERBAN, E. 1973. Sur les Bathynellidae (Podophallocarida Bathynellacea) de l'Italie: Sardobathynella cottarellii n.g. et n. sp. Fragm. Ent., IX, 2, 81-107.

SERBAN, E. 1973a. Sur les Bathynellidae (Podophallocarida, Bathynellacea) de l'Italie: Bathynella ruffoi nov. sp. et Bathvnella lombardica nov. sp. Mem. Mus. Civ. St. Nat. Verona, XX, 17-37.

SERBAN, E. 1973b. Sur les problèmes de la taxonomie des Bathynellidae (Podophallocarida Bathynellacea). Livre du cinquantenaire de l'Institut de Spéologie "Emile Racovitza". Ed. Acad. de la R.S.R., Bucarest, 199-217.

SERBAN, E. 1973c. Sur le processus de la pléonisation du péréion dans l'ordre des Bathynellacea (Crustacea, Malacostraca, Podophallocarida). Biidr. Dierk., XXXIII, 2, 173-201.

SERBAN, E., COINEAU, N. \& DELAMARE DeBOUTTEVILle, CL. 1971. Les Gallo- 
bathynellinae, nouvelle sous-famille des Bathynellacea (Crustacea, Malacostraca). C. R. Acad. Sci., CCLXXII, 2907-2909.

SERBAN, E., COINEAU, N. \& DELAMARE DEBOUTTEVILLE, CL. 1972. Recherches sur les crustacés souterrains et mésopsammiques. Les Bathynellacés (Malacostraca) des régions méridionales de l'Europe Occidentale. La sous-famille des Gallobathynellinae. Mem. Mus. Sci. Nat. Paris, LXXV, 1-107.

SERBAN, E. \& DELAMARE DEBOUTTEVILLE, CL. 1974. Contribution à l'étude de Bathynella chappuisi Delachaux (Bathynellacea Bathynellidae) Ann. Spéléol., XXIX, 1, 77-96.

SERBAN, E. \& COINEAU, N. 1975. Sur les Bathynellidae (Podophallocarida, Bathynellacea) d'Afrique du Sud: 1. Les genres Transvaalthynella nov. et Transkeithynella nov. Ann. Spéléol. XXX, 137-165.

VEJDOVSKY, FR. 1882. Thierische Organismen der Brunnenwässer von Prag. Prag, 1-70. 